BULL. AUSTRAL. MATH. SOC.

\title{
THE TIME DEPENDENT \\ NET MATERNITY FUNCTION
}

\section{Pietro Cerone}

This thesis examines the resultant behaviour of a population in response to changes of the age-specific birth and death rates with time. The deterministic one-sex population model of Sharpe and Lotka is used as the basis for the analysis. In particular, the asymptotic behaviour is determined for a population with a time dependent net maternity function. Thus, the present study may be looked upon as representing a generalisation of stable population theory to include models of time dependent vital rates of birth and death. Laplace transform techniques are used extensively throughout the present work.

The problem of Keyfitz on the momentum of population growth is generalised to contain a gradual exponential scaling (at a rate $\lambda$ ) of the age-specific birth rate to the level of bare replacement. An algorithm for obtaining the asymptotic total birth rate for general initial net maternity functions is outlined. The method is evaluated by comparing known analytic asymptotic values for two simple initial net maternity functions, to the approximations obtained through the algorithm. The converse problem is also examined: given a prescribed asymptotic population level, it is desired to determine the transition rate $\lambda$, which characterises the change of the age-specific birth rate. The converse problem is important in the planning and management of populations.

An extension of the recurrence relation method on which the above algorithm was based, enables a description of the transient behaviour of

Received 3 January 1980. Thesis submitted to the University of Wollongong, May 1979. Degree approved December 1979. Supervisors: The late Professor A. Keane, Dr T.S. Horner. 
the population. Short of using a strictly numerical method for solving the integral equation governing the total birth rate, the transient behaviour may also be obtained by a stepping procedure when the age structure of the time dependent net maternity function is defined in a piecewise fashion. Models are also proposed which allow for a time dependent change of the initial net maternity function more general than the simple exponential. The asymptotic behaviour of the ensuing population is evaluated. 